\title{
La gestión directiva: un concepto construido desde las comprensiones de los directivos docentes de las escuelas públicas bogotanas
}

The directive management: a concept derived from the understandings of principals and coordinators at public schools in Bogota

A gerência executiva: um conceito construído a partir das percepções dos professores executivos em escolas públicas Bogotá

Sorangela Miranda Beltrán

Universidad Pedagógica Nacional, México mirandasorangela@gmail.com

\section{Resumen}

El presente artículo, recoge los principales referentes teóricos y la construcción conceptual sobre la categoría gestión directiva, surgida del trabajo investigativo desarrollado en el postdoctorado en Administración y Gestión de Planteles Educativos del Centro de Estudios e Investigaciones para el Desarrollo Docente CENID.

La investigación tuvo como objetivo central la construcción conceptual sobre la categoría "gestión directiva", a partir de las significaciones, los sentidos y las comprensiones que desde las prácticas pedagógicas y administrativas, le dieron los directivos docentes participantes del estudio (rectores y coordinadores) de tres escuelas públicas ubicadas en Bogotá- Colombia.

Siendo así, este documento se presenta como una construcción conceptual que puede ser contrastada, comparada y enriquecida con otras experiencias en este campo, procurando encontrar con ello diversos elementos que, desde las perspectivas empírica y teórica, establezcan nuevos horizontes para la comprensión de la categoría gestión en el contexto educativo y muy particularmente, desde la mirada de los directivos docentes de la escuela pública, como un fenómeno complejo y de amplio debate en el mundo actual. 
La apuesta metodológica se centro en la construcción propia un trabajo investigativo, que comprendió las componentes epistemológica (desde la hermenéutica, la crítica y participación de quienes hacen parte del proceso) y Ontológica (partiendo de las apuestas por el sujeto conocido), como ejes de trabajo para la construcción de un concepto de gestión desde los conocimientos y la praxis de los maestros, contribuyendo en transformar e innovar su quehacer en la escuela.

Palaras Clave: Gestión directiva, directivos-docentes, escuela, administración educativa, direccionamiento, liderazgo, comunicación.

\section{Abstract}

This article gathers the main theoretical constructs and the conceptual construction about the category directive management that emerges from the research work in the post doctorate in Administration and Management of Educational Institutions at Centro de Estudios e Investigaciones para el Desarrollo Docente CENID.(Center of Studies and Research for the Teacher Development)

The main objective of the research was the conceptual construction about the category "directive management". It arose from the significances, meanings and understandings that the participant teachers (principals and coordinators from three public schools in Bogota Colombia) constructed from their pedagogical and administrative practices.

This document is a conceptual construction that can be contrasted, compared and enriched with other experiences in this field. It tries to find diverse elements from empirical and theoretical perspectives that establish new horizons for the understanding of the category management in the educational context and mainly from the view of the principals and coordinators at public schools as a complex phenomenon with large debate in the current world.

The methodological design focused on the specific construction of a research work that comprised the epistemological components (from the hermeneutics, critical aspects and the participation of the ones involved in the process) and ontological (starting from the understandings of the known subject) as axes for the construction of a concept of 
management from the knowledge and reflexive practices of teachers as a way to contribute in the transformation and innovation in their daily work at schools.

Key words: directive management, principals and coordinators, school, educational administration, addressing, leadership, communication.

\section{Resumo}

Este artigo resume o principal referencial teórico e construção conceitual na categoria de gestão política, decorrente do trabalho de investigação realizado na pós-doutorado em Administração e Gestão de instituições educacionais do Centro de Estudos e Investigação Faculdade CENIDs Desenvolvimento.

A pesquisa teve como objectivo central a construção conceptual da categoria "gestão executiva" de significados, sentidos e entendimentos que, desde o pedagógica e as práticas administrativas, deu professores participantes diretores do estudo (reitores e coordenadores) de três escolas públicas de Bogotá Colômbia.

Assim, este documento é apresentado como uma construção conceitual que pode ser testada, em comparação e enriquecido com outras experiências neste campo, tentando encontrar isso várias elementos das perspectivas empíricas e teóricas, estabelecer novos horizontes para a compreensão categoria gestão no contexto educacional e, particularmente, a partir da perspectiva dos gestores de professores da rede pública, como um fenômeno complexo e amplamente discutido no mundo de hoje.

A metodologia aposta centrada na própria construção trabalho de investigação, que incluiu os componentes epistemológicos (de hermenêutica, a crítica e participação daqueles que são parte do processo) e Ontologia (baseado em apostar o assunto conhecido) como eixos Eu trabalho para a construção de um conceito de gestão do conhecimento e prática dos professores, ajudando a transformar e inovar o seu trabalho na escola.

Palaras chave: Os directores-gerais, diretores de escola, escola, administração educacional, abordando, liderança, comunicação. 


\section{Introducción}

En las últimas décadas, la profesión de enseñar se ha visto inmersa en una serie de paradojas que apuntan a la exigencia para los directivos docentes de llevar a sus instituciones acorde con los requerimientos que el mundo global les impone en tensión con la urgencia de sortear el día a día que recae en la escuela y que, sin duda alguna, representa un cumulo de realidades problémicas para quien tiene la tarea de administrar este tipo de instituciones: las situaciones derivadas de las fracturas sociales, la violencia escolar y los avances en la sociedad del conocimiento, son algunas de las variables que se muestran complejas a la hora de gestionar en las organizaciones educativas.

De esta manera, el directivo docente, cargado de múltiples retos y exigencias sociales, se ve inmerso en una dinámica donde su rol se reduce a la ejecución operativa de sus funciones como una tarea que garantiza el control y la existencia de la institución que dirige, en el marco de las intencionalidades de la oferta y la productividad económicas que generan discursos y políticas que desdibujan el lugar político, pedagógico y social del directivo docente y lo ubican en un escenario de tensiones y demandas propias de un sistema que impone un funcionamiento lineal y no pensante de la escuela.

Desde esta óptica el directivo docente, es el "trabajador", cuya función es inherente al desarrollo orgánico de la sociedad (Gramsci, 1972), siendo invisibilizado en la historia, silenciado en la reflexión, cuestionado en su ejercicio, separado del territorio del saber pedagógico (Noguera, 2005), arrancado de todo intento por pensarse a sí mismo como un intelectual con posibilidad para trasformar y generar nuevos conocimientos y dinámicas en la gestión y hacer de su praxis pedagógica y administrativa, un interesante campo para la innovación, la investigación y el crecimiento epistemológico. 
Ambas consideraciones, la marginalidad intelectual del directivo docente y la concepción instrumental de la gestión directiva, posibilitaron un acercamiento inicial a la pregunta sobre las comprensiones que estos actores institucionales tienen sobre la gestión en sus escuelas, tensionando dos interesantes variables: por un lado, el reconocimiento de los directivos como sujetos válidos en las interacciones cognitivas, por el otro, la apertura hacia una visión de la gestión directiva significada desde los saberes y las realidades de quienes la desarrollan en las organizaciones educativas escolares.

Con estos primeros elementos de análisis, enriquecidos en el diálogo con los rectores y los coordinadores de las escuelas públicas bogotanas que participaron voluntariamente en la investigación, se hizo posible profundizar el campo temático a estudiar, en la medida en que, a través de la interacción con los directivos docentes en el diálogo directo con ellos, en el acompañamiento in situ y en espacios de discusión pedagógica, ha hizo posible retomar sus perspectivas, intereses, preocupaciones e inquietudes para integrarlas en un todo y retomarlas en este documento.

Para ello, en primer lugar, se referirá el trabajo teórico que comprendió el estudio, para luego enfatizar en las visiones de los directivos docentes y el significado que para ellos tiene la gestión directiva en sus colegios.

\section{La teoría como punto de partida para la construcción del concepto de gestión desde las comprensiones de los directivos docentes}

Las consideraciones teóricas que se enunciaran a continuación, se constituyeron en los preconceptos con los cuales como el sujeto cognoscente (investigadora) se acerco al sujeto conocido (directivo-docente) en el contexto específico de este estudio ${ }^{1}$, en un intento por

\footnotetext{
${ }^{1}$ Siguiendo a Vasilachis (2003), el sujeto conocido en la investigación, fue representado en el directivo docente, quien se asumió como sujeto autorreferente para la construcción de conocimientos desde su experiencia, los saberes y las comprensiones sobre su realidad. El sujeto cognoscente (quien investiga), es ubicado por Vasilachis (2003) en un rol donde existe la posibilidad no sólo de transformar, sino también de aprender de los otros, crecer en la interacción y desarrollar la capacidad para conocer a los demás, valorando sus emisiones, experiencias y visiones de mundo. La realidad construida desde la perspectiva colectiva, le permite a quien investiga utilizar diversos métodos de acercamiento a la misma, enfatizando en la participación, el diálogo, la lectura de datos que se construyen y tienen lugar en el escenario contextual de quienes hacen parte del proceso.
} 
construir una primera noción conceptual sobre la gestión directiva que orientó la comprensión de la realidad que se busco profundizar.

En esta construcción teórica, se enfatizo en la capacidad para entender la relación entre los saberes y la experiencia de los directivos docentes, con una actitud posibilitadora de cambios y visionaria ante los desafíos de la gestión de las organizaciones educativas en el mundo actual, indagando en primera instancia por el concepto de gestión que se desarrolla a continuación:

\subsection{El concepto de gestión}

Las ideas que se desarrollarán en los siguientes apartes, son producto de un trabajo arduo de investigación de Tabares y Miranda (2015), quienes se encargaron de contextualizar la categoría gestión directiva en campo de las escuelas públicas bogotanas, ubicando esta categoría en el terreno de la administración en educación, estableciendo al interior de este concepto sus elementos y relaciones. El planteamiento de estos autores se especifica a continuación:

\subsubsection{La Gestión Directiva y la Administración Educativa}

En el contexto colombiano, el Ministerio de Educación Nacional - MEN- (2008), define los elementos que permiten caracterizar la gestión en las organizaciones educativas escolares, asumiendo este concepto desde cuatro campos de acción:

- La Gestión Académica: Que se encamina en lograr que los estudiantes aprendan y desarrollen las competencias necesarias para su desempeño personal, social y profesional, retomando los procesos de diseño curricular, prácticas pedagógicas institucionales, gestión de clases y seguimiento académico.

- La Gestión Administrativa y Financiera: Área en la que se realizan los procesos de apoyo a la gestión académica, la administración de la planta física, los recursos y los servicios, el manejo del talento humano y la parte financiera y contable. 
- La Gestión de la Comunidad: Referida a las relaciones de la institución con la comunidad, la participación y la convivencia, la atención educativa a grupos poblacionales con necesidades especiales.

- La Gestión Directiva: Área se centra en el direccionamiento estratégico, la cultura institucional, el clima y el gobierno escolar, además de las relaciones con el entorno. De esta forma, es posible que el rector o director y su equipo directivo organicen, desarrollen y evalúen el funcionamiento general de la institución.

\subsubsection{Teorización de la gestión directiva y sus principales procesos:}

A partir de lo expuesto y siguiendo los lineamientos del Ministerio de Educación en Colombia, se encuentran los siguientes procesos que hacen parte de la gestión entendida desde el ámbito de la escuela:

\section{- El Direccionamiento Estratégico y el Horizonte Institucional}

Es el componente base alrededor del cual giran los valores, principios, la misión, la visión y la filosofía de los establecimientos educativos y se materializa en un elemento fundamental: el Proyecto Educativo Institucional (PEI).

El artículo 73 de la Ley 115 de 1994, señala que el Proyecto Educativo Institucional es el conjunto de principios y fines del establecimiento, los recursos docentes didácticos disponibles y necesarios, la estrategia pedagógica, el reglamento para docentes y estudiantes y, el sistema de gestión, encaminado a responder a situaciones y necesidades de los educandos, de la comunidad local, de la región y del país, de manera concreta, factible y evaluable.

La trascendencia del PEI para una organización escolar, se materializa en su cultura, los valores que la fundamentan y las acciones de todos los miembros de la comunidad educativa, quienes, siguiendo los principios del horizonte institucional, configuran las acciones que integran las gestiones académica y administrativa. 


\section{- La Gestión Estratégica y la Visión del Liderazgo}

Para Lana (2014, citado por Tabares y Miranda, 2015), la Administración Estratégica constituye un proceso continuo e interactivo que se implementa para mantener a la organización como un todo integrado con su ambiente, planteamiento que sugiere un trabajo directivo en el cual se privilegien las capacidades de liderazgo y el fortalecimiento de la comunicación institucional, como elementos necesarios para encaminar la organización escolar hacia la consolidación de acciones conjuntas que le permitan planear coordinadamente los procesos para el alcance de los objetivos, asumiendo una visión prospectiva ante las exigencias del entorno cambiante.

De manera transversal en la gestión directiva, según Tabares y Miranda (2015), el liderazgo emerge como uno de los aspectos más importantes que en la actualidad requiere toda organización, convirtiéndose en una característica necesaria para quienes integran los equipos de dirección, reconociendo que el directivo docente, además de cumplir con las tareas administrativas propias de su cargo, debe combinar el "Liderazgo dentro de su proceso de gestión, donde sus competencias, habilidades y actitudes sirvan de apoyo para desarrollar su desempeño profesional y esté en capacidad de influir, dirigir y motivar a su equipo de trabajo, orientándolo hacia la consecución de metas compartidas a través de una mejora permanente de rendimiento” (Rincón, 2011. Pág. 21).

De esta manera, quién asume el reto de liderar instituciones educativas, debe saber potenciar el talento humano, planear el trabajo en áreas estratégicas, orientar hacia la consecución de los objetivos y las metas establecidas en el PEI y coordinar las acciones de seguimiento, evaluación y retroalimentación de los diversos procesos, propiciando dinámicas de diálogo, trabajo en equipo y construcción colectiva en los diferentes estamentos de la comunidad educativa. 


\section{- La comunicación organizacional como estrategia para la gestión}

Siguiendo a Tabares y Miranda (2015), la comunicación penetra a todas las actividades de una organización. Es un proceso que se define como la posibilidad de "Crear valor a través de la transmisión y/o recepción de un mensaje con contenido" (Gorrochotegui, 2010. Pág. 45), representando una importante herramienta de trabajo a través de la cual, los individuos entienden su papel en la entidad (Everett y Rekha, 1980, citado por Zapata, 2008), generando la afluencia de relaciones coordinadas en las diferentes áreas organizacionales para el alcance de las metas estratégicas.

De este modo, dentro de las dinámicas propias de las instituciones educativas, la comunicación posee un carácter vital, caracterizándose por ser el medio regulador de las interacciones que subyacen en los diversos procesos de gestión, asumiendo la forma como, en los escenarios comunicativos, la correlación entre los equipos y grupos de trabajo se configura en las interacciones, planteamiento evidenciado en el pensamiento de Gorrochotegui (2010) al expresar que: "La relación entre los miembros de un equipo es de interdependencia y de colaboración y requiere unidad de criterio y de actuación. Por este motivo, es frecuente que aparezcan situaciones de conflicto que es necesario manejar. Para ello, se requieren normas específicas de comunicación, de modo que los miembros del equipo tengan expectativas claras y compartidas en todo momento" (Gorrochotegui, 2010. Pág. 55).

\section{- Gobierno Escolar y las formas de participación en el ámbito escolar}

El Gobierno Escolar es el mecanismo para garantizar la representación de los diferentes estamentos de la Comunidad Educativa en las discusiones y decisiones que afectan a la organización escolar (Secretaría de Educación Distrital-SED-, 2013), legitimando las formas de participación y democracia que caracterizan las nuevas propuestas en la gestión de las organizaciones educativas. En este proceso, los órganos de Gobierno Escolar y las demás instancias de participación, son los elementos privilegiados en el análisis y la construcción teórica realizada en esta investigación. 


\section{Los órganos de Representación de la Comunidad Educativa}

Guevara (2006), otorga a los representantes de la Comunidad Educativa, una responsabilidad dada por el poder de los estamentos del colegio que los elige para ejercer autoridad legítima a través de las funciones que les atañen, mediante unas decisiones que buscan proteger los derechos de los representados.

Estos órganos de representación escolar en el contexto colombiano, están definidos por la Ley General de Educación (Ley 115 de 1994), en su artículo 142, donde se establece que hacen parte del Gobierno Escolar, el rector o la rectora -como representante legal de la institución educativa, ejecutor o ejecutora de las decisiones del Consejo Directivo y responsable de la organización ante los entes que ejercen el control del servicio educativo-, el Consejo Directivo -máxima instancia para la toma de decisiones que afectan el funcionamiento de la institución, excepto las que sean competencia de otra autoridad (Guevara, 2006. Pág. 96).- y el Consejo Académico- como órgano asesor en todo lo curricular, lo pedagógico y lo formativo de cada institución, que, entre otras funciones, desempeña la de servir de consultor del Consejo Directivo en la revisión del PEI (Guevara, 2006. Pág. 103).

\section{Otras Instancias de Participación de la Comunidad Educativa}

En las organizaciones educativas escolares en Colombia, según el Decreto 1860 de 1994 se encuentran las siguientes estancias de participación de la Comunidad Educativa en los diversos procesos institucionales:

a. El personero o la personera de los estudiantes, un o una estudiante del último grado ofrecido por la institución, cuyo compromiso se orienta en promover el ejercicio de los deberes y derechos de los demás niños, niñas y jóvenes del colegio según la Constitución Nacional y el Pacto o Manual de Convivencia.

b. El Consejo Estudiantil, como máximo órgano colegiado que asegura y garantiza el continuo ejercicio de la participación por parte de los niños, niñas y jóvenes del colegio en las decisiones que los atañe, teniendo como vocero en el Consejo Directivo, al 
estudiante del último grado ofrecido por la organización escolar.

c. La asociación de padres de familia, como ente autónomo con personería jurídica que contribuye en actividades y acciones para el buen desarrollo del PEI.

d. El Consejo de padres de familia, como instancia para asegurar la continúa participación de los padres y acudientes en el proceso pedagógico de la organización escolar.

- La Cultura Institucional: el sistema de valores de las Comunidades Educativas

Una mirada conjunta hacia los propósitos de la organización, constituye un importante reto para los equipos directivos, quienes tienen la tarea de armonizar el cambio de representaciones sociales (Tapiero y López, 2006 citado por Tabares y Miranda, 2105), que emergen en un sin número de individualidades que se articulan y a quienes se debe liderar para desarrollar acciones colectivas en aras de los objetivos propuestos.

Lograr la participación de los diferentes elementos que conforman la entidad y en especial, el equilibrio entre ellos, requiere la implementación de una metodología, que más allá de convertirse en un ejercicio mecánico o impositivo por parte de las directivas, se constituya en eje motivador para crear visiones conjuntas, entendiéndose esta metodología como "Una combinación de objetivos, de técnicas, de tiempos, de locales, de material escrito a estudiar, de descansos, de cambios de velocidad y ritmo en el desarrollo de cada sesión, de flexibilidad y de exigencia, de permisibilidad y capacidad de conducción" (Álvarez, 2003. Pág. 93).

La calidad, la eficacia y la eficiencia del trabajo en equipo, dependerá de la componente humana, es decir, de la formación y experiencia de los participantes, del interés y atractivo que ofrezcan los objetivos, de la integración en el trabajo, del sentido de responsabilidad de quienes conforman la organización (Álvarez, 2003) y del liderazgo del directivo, cuyo desafío está representado en propender por formas participativas que generen sentido de pertenencia y compromiso institucional para el buen avance de los procesos. 


\section{- Clima Organizacional para el éxito de la gestión directiva}

Continuando con los planteamientos de Tabares y Miranda (2015), el clima organizacional es la calidad del ambiente de la organización que es percibido o experimentado por sus miembros y que influye ostensiblemente en su comportamiento, "Se constituye en un medio facilitador/obstaculizador de los resultados, cuanto por contribuir él mismo a los efectivos logros del centro" (Ruiz, 2004. Pág. 33) y asume diversas connotaciones que pueden ser favorables o no para los miembros de la organización: favorable cuando satisface las necesidades personales de los participantes y eleva su estado de ánimo, desfavorable cuando despierta la frustración y no se satisfacen tales necesidades (Chiavenato, 2005).

Dentro del clima organizacional, se pueden destacar dos subprocesos que lo integran: el tratamiento de los conflictos y los entornos de aprendizaje, los cuales se exponen a continuación:

La resolución de Conflictos: En el trabajo con grupos humanos, la complejidad de las relaciones, las características individuales en interacción con las de los demás sujetos, puede convertirse en un entramado de encuentro de saberes y/o de diferencias, acorde con lo expuesto por Gorrochotegui (2010), para quien, la interacción entre las personas lleva implícita la posibilidad de conflicto, el cual permanece latente o puede aparecer explícitamente.

La negociación: Para Chiavenato (2005), la negociación es un proceso mediante el cual, dos o más partes intercambian valores. Para este autor, los enfoques de la negociación pueden ser tradicionales (negociación, distributiva por medio de canjes de distribución, o de canjes de posiciones) o modernas (negociación integradora, basada en las habilidades para negociar) e incluye procesos tales como la preparación y el plan de acción, definición de las reglas básicas para negociar, las aclaraciones y justificaciones, los canjes y las fechas de aplicación o cumplimiento de los compromisos pactados. 
Los entornos de Aprendizaje: Para una organización educativa, es fundamental pensar en los entornos de aprendizaje como parte de su cultura y su clima organizacional, toda vez que la naturaleza de sus acciones giran en torno al fomento de las capacidades de los diversos miembros de la Comunidad Educativa (en especial la población escolar) para adquirir nuevos conocimientos, modificar hábitos y estar abiertos al cambio (Gorrochotegui, 2010).

\section{- Comunidad Interna y Externa como contextos en la dirección escolar}

Tabares y Miranda (2015) manifiestan que en la gestión directiva, los procesos que se desarrollan con la comunidad pueden enmarcarse en tres grandes aspectos: la normatividad educativa vigente que regula todos los procesos llevados a cabo desde la administración del centro escolar, las relaciones entre la escuela y el sector productivo como ejes articuladores del impacto social de la organización en los ámbitos local y regional y los procesos de evaluación permanente, como posibilidades para el mejoramiento y la consolidación de las prácticas directivas exitosas. Revisemos a continuación cada uno de estos aspectos:

La Normatividad educativa vigente: La gestión directiva tiene como una de sus importantes responsabilidades, acatar y llevar a cabo las políticas públicas vigentes en materia educativa. Cualquiera que sea el modelo por el cual se opte a la hora de administrar la organización escolar, la normatividad en educación marca los rumbos del direccionamiento estratégico y los propósitos mismos del PEI.

Escuela y sector productivo: Siguiendo lo expuesto por el Ministerio de Educación Nacional- MEN- (2003), en lo relacionado con la educación y el sector productivo, esta entidad define "el mundo del trabajo como un escenario fundamental para el desarrollo personal de los individuos", siendo este un elemento esencial para la construcción de los proyectos de vida de quienes se forman en las escuelas. 
Para el MEN, prepararse para el mundo del trabajo, implica no sólo la vinculación al sector laboral, sino también el potenciar habilidades, capacidades y destrezas para generar autoempleo, empresas o unidades asociativas, acorde con las exigencias del mundo actual, en el que cada día se hace necesario contar con conocimientos y saberes para proponer de cara a la productividad y la competencia.

Los procesos de evaluación permanente: En este proceso, la labor directiva debe encaminarse en que "El establecimiento educativo recoja, sistematice, analice y valore la información para compararla con el desarrollo de sus acciones y resultados en las diversas áreas de la gestión institucional (directiva, académica, administrativa y comunitaria), lo que le facilita identificar las fortalezas y debilidades en su funcionamiento y le sugiere correctivos y planes de mejoramiento" (Colombia Aprende, 2014).

Entendida así, la evaluación se convierte en un recurso permanente que garantiza el cumplimiento de los propósitos organizacionales, posibilitando acciones permanentes de mejora y ajuste de los procesos, de manera coherente con los indicadores y las metas que la entidad prevé en sus Planes de Mejoramiento.

\section{Método: Construyendo la experiencia. El concepto de gestión desde los directivos docentes.}

Para construir el concepto de gestión desde la voz de los directivos-docentes participantes en esta investigación, se llevo a cabo un trabajo directo en las escuelas, para acercarse al sujeto conocido y retomar con ellos sus preocupaciones, intereses y experiencias administrativas como aspectos fundamentales para ampliar las nociones sobre esta categoría.

Para ello, se opto por centrar la atención en la localidad séptima de Bosa en BogotáColombia, extendiendo la invitación a través del contacto personal a tres rectoras y sus equipos de coordinadores de las instituciones educativas de esta zona, toda vez que con ellos se ha venido adelantando otro tipo de procesos de índole académica y administrativa, 
que durante estos años han permitido conocerlos y conocer sus instituciones en lo referente a sus características, problemáticas y alcances en la comunidad local.

Las rectoras invitadas aceptaron ser participes de esta investigación en los tiempos establecidos para ella e invitaron a sus directivos a que participaran del estudio. De un total de 11 coordinadores, 8 voluntariamente quisieron hacer parte de este proceso.

Las rectoras fueron fundamentales en cada una de las fases y etapas del trabajo de campo, contribuyendo con la apertura de los tiempos y espacios necesarios para su desarrollo. Con el apoyo de estas directivas, se lograron dos propósitos fundamentales: el primero, el diálogo directo con cada uno de los coordinadores para invitarlos a hacer parte de la investigación y el segundo, el acceso a los documentos que dan cuenta de los ejercicios de gestión en cada una de las instituciones educativas.

El desarrollo del diseño metodológico propuesto en la tarea investigativa implico una serie de decisiones metodológicas derivadas de los conocimientos y saberes del sujeto cognoscente (las rectoras y coordinadores participantes) y los intereses y objetivos de la investigación, en un ejercicio de creatividad en el abordaje de los datos propio de quien investiga con curiosidad, motivación, deseo por indagar, profundizar y lograr comprensiones desde la perspectiva de quienes se pretende conocer.

De este modo, la delimitación del universo de datos recogidos en este proceso investigativo, atendió a lo expuesto por Glaser y Strauss (1967), en cuanto a la relevancia en la definición de criterios teóricos que permitieran el rastreo de información relevante que contribuyera a dar respuesta a la pregunta central que ocupa la atención en este estudio.

Las observaciones realizadas y las entrevistas se estructuraron alrededor de cuatro categorías coherentes con los objetivos general y específicos de esta investigación encaminándose a la identificación sobre las comprensiones y la caracterización que los directivos docentes hacen del concepto sobre gestión en sus colegios, revisando a la vez las habilidades, conocimientos y capacidades que debe tener un directivo para ejercerla, el 
papel que desempeñan en las instituciones educativas y las implicaciones de estos aspectos en sus prácticas administrativas.

Es así, como a partir de los objetivos del estudio, se definieron las categorías estructurales que concretaron los propósitos de la investigación y sirvieron como directriz para responder a la pregunta central propuesta: El concepto sobre la gestión desde las comprensiones de los sujetos conocidos, la caracterización de la gestión directiva desde la mirada de los directivos-docentes, la formación profesional y las praxis directivas en la construcción del concepto de gestión y el rol de los directivos docentes en la gestión de las instituciones educativas. Los resultados de este trabajo se especifican a continuación:

\section{Resultados: El concepto sobre la gestión desde las comprensiones de los sujetos conocidos}

Construir conceptualmente la categoría gestión directiva a partir de las significaciones, los sentidos y las comprensiones que desde las prácticas pedagógicas y administrativas le dan los directivos docentes (rectores y coordinadores) de las Escuelas Públicas Bogotanas, es el propósito fundamental de este estudio y el pretexto para acercar a los sujetos conocidos a un diálogo de saberes caracterizado por las reflexiones y la puesta en escena de los conocimientos que emergen de su trabajo cotidiano en las escuelas.

Para los directivos participantes, la gestión en sus colegios comprende los diversos procesos encaminados a facilitar la coordinación de una organización, constituyéndose en un conjunto de acciones orientadas a la consecución de objetivos y propósitos de la entidad, los cuales se materializan a través de sus Proyectos Educativos Institucionales (PEI) y se hacen operativos ejerciendo las tareas directivas dentro y fuera de la organización por parte un equipo que los oriente y las controle para lograr el éxito pedagógico y convivencial.

Gestionar es entonces, un ejercicio de actitud sumado a capacidades pedagógicas y administrativas de quien dirige, de tal manera que se puedan alcanzar las transformaciones significativas en el colegio. Es desarrollar engranaje un proceso educativo donde se profundiza en el contexto de los estudiantes desde la realidad de los actores visto a partir 
del desarrollo de los procesos que permite direccionar estratégicamente los ideales de la institución.

Así, la gestión directiva es el conjunto de acciones encaminadas a la consecución de los objetivos y propósitos de una entidad que nace de un ejercicio propio de la administración y se enfoca en facilitar la coordinación de una organización, como una tarea se ejerce dentro (contexto interno) y fuera de la institución (contexto externo), por parte del equipo directivo y que orienta controla todas las actividades curriculares y extracurriculares, procurando el éxito y la calidad del quehacer pedagógico y convivencial.

La relación que se establece entre esta definición de la gestión y la tarea directiva, se articula entre el ser y el hacer, que significa liderar procesos y procedimientos que se hacen transversales en los planos convivencial, académico y administrativo, acorde con las funciones que se desarrollan y que implica planear, evaluar y controlar todos los procesos de la institución, dependiendo de sus características y contribuyendo en desarrollar de manera eficaz y eficiente dichos procesos.

Los equipos directivos cumplen un papel fundamental en estos procesos, dado que son ellos los llamados a proponer estrategias de mejoramiento para el alcance de las metas en concordancia con la misión, visión y horizonte institucional a través de la apropiación de los objetivos corporativos y el diseño y ejecución de las acciones pertinentes, tales como la proyección anual, la evaluación de procesos, la planeación de acciones para el mejoramiento, el diseño de planes para el aprovechamiento de los recursos institucionales y el fortalecimiento de los valores que promueve el colegio como parte fundamental para la consolidación de su cultura institucional.

Desde la práctica pedagógica y administrativa, la gestión directiva es relevante para quienes la ejercen, en la medida en que contribuye en mantener la mirada puesta en los horizontes institucionales, supervisar las acciones que se ejecutan, permite redireccionar las estrategias y hace necesario estar atento a las diferentes áreas comprometías en cada uno de los procesos, interpretando y profundizando los contextos para direccionar la administración de la organización. 
La gestión directiva es importante porque permite cumplir las metas y todo lo que concierne al horizonte institucional para el mejoramiento colectivo a partir del trabajo mancomunado de los equipos directivos, quienes tienen la responsabilidad de que la misión, visión y objetivos de la institución se cumplan. En estos equipos, su liderazgo pedagógico en los procesos y procedimientos y la supervisión, son aspectos esenciales y deben darse acorde con las funciones de cada directivo en las actividades que se desarrollan diariamente en los colegios.

Como un aspecto relevante en el concepto sobre la gestión que hacen los sujetos conocidos en este estudio, se contempla la comunicación asertiva para poder realizar seguimiento a los procesos tendientes al fortalecimiento de la institución en la implementación y seguimiento de proyectos, planes y programas de carácter académico, administrativo y convivencial que se lleven a cabo en beneficio de los estudiantes y su formación integral.

Para los directivos es importante mantener la mirada puesta en los horizontes institucionales, supervisar las acciones que se discutan, redireccionar las estrategias si se hace necesario y estar atentos en las diferentes áreas comprometidas en el proceso, la gestión atendiendo a la interpretación y el valor de los contextos para orientar las tareas pedagógicas de enseñanza y aprendizaje.

Dentro de este concepto de gestión directiva, se deben cumplir las metas y todo lo que concierne al horizonte institucional (visión, misión, objetivos, filosofía y estrategias institucionales de mejoramiento), todo dentro de la responsabilidad colectiva vista como una unidad de acción y alineamiento del equipo directivo dado que, independientemente de las capacidades de todos los que trabajan en una institución educativa, se necesita el liderazgo de un equipo que direccione las fortalezas y oportunidades de los demás en torno al PEI.

Teniendo en cuenta lo expuesto, los directivos docentes participantes en esta investigación resaltan que al referir la categoría gestión en la educación, se hace mención a un proceso que involucra el desarrollo de todas las actividades educativas tendientes al mejoramiento de la calidad de los procesos pedagógicos, académicos, administrativos, convivenciales y 
comunitarios, respondiendo al escenario legal o las políticas en materia de educación en el país, como foco que imparte las pautas para que la organización se adecúe y responda al sistema educativo.

Al coordinar las directrices y campo de acción de cada estamento de la institución hacia la consecución de los objetivos propuestos que permitan la formación integral de la comunidad estudiantil y el desarrollo y consolidación de su propia entidad como institución educativa, la gestión directiva toma forma hacia una visión compartida que hace plausible la puesta en marcha de proyectos y planes para orientar a los miembros de la comunidad hacia los fines propios del colegio, reconociendo en este ejercicio los momentos del diagnóstico, planeación, ejecución, evaluación y seguimiento.

De esta forma, la gestión en educación para los sujetos conocidos de esta investigación, es desarrollar todas las actividades educativas tendientes al mejoramiento de la calidad en el servicio de enseñanza, gestionando entre otros, un currículo que fortalezca el proyecto de vida de los estudiantes, atendiendo a la política pública educativa y promocionando la formación integral de los niños, niñas y jóvenes dentro de los parámetros de la calidad y la pertinencia.

\section{Discusión:}

\subsection{La caracterización de la gestión directiva desde la mirada de los directivos-docentes}

La gestión educativa a diferencia de otros campos de la gestión, se encamina hacia la formación integral de seres humanos, siendo la persona el factor primordial para transformar la sociedad a partir del desarrollo de tareas educativas que fomenten en conocimiento sobre sí mismo y la capacidad de actuar en ciudadanía y equilibrio con el entorno.

La gestión educativa se encuentra orientada hacia las personas en busca el mejoramiento y crecimiento del ser humano, partiendo de sus características antropológicas y reconociendo a la mujer y al hombre en su humanidad y el crecimiento integral de quienes se forman en las escuelas. Esta gestión, se diferencia de la gestión en general porque los fines que persigue se encaminan a la formación de ciudadanos y ciudadanas para la vida, el mundo y 
su pleno desarrollo como personas en un contexto que procura contribuir al desarrollo social.

Dentro de esas características, también se encuentra el entender la administración y la gestión como ejercicios desarrollados por las personas para las personas, orientando el talento humano al manejo y aprovechamiento de recursos físicos y materiales, dentro de responsabilidades institucionales que involucran el trabajo en equipo, la toma decisiones, la apertura a la innovación, la lectura clara de los contextos humanos y sociales, la capacidad de escucha para la solución de conflictos, la búsqueda del bien común y la puesta en marcha del PEI.

Con base en estas características, se pueden determinar una serie de habilidades, conocimientos y capacidades que tiene que ver con el perfil del directivo docente para desenvolverse en este complejo concepto de gestión directiva desde la perspectiva humanística. Dentro de estos aspectos, el directivo debe tener liderazgo positivo, una visión humana que le permita comprenderse y comprender a sus interlocutores, manejo teórico en gestión y administración educativas, capacidad pedagógica y de investigación, contando también con capacidad de direccionar estratégicamente a la organización, haciendo uso de sus capacidades para la comunicación asertiva y siendo facilitador y conciliador en los diferentes momentos y espacios que se viven en la gestión de las instituciones.

Debe ser un líder propositivo con convicción de su vocación, habilidades y competencias comunicativas ética y profesionalismo, actitud asertiva propositivo en su accionar, comprometido con responsabilidad, autodominio, inquieto por saber y recursivo.

El directivo docente debe ser una persona capaz de gerenciarse a sí mismo, entendido esto como la capacidad de auto evaluarse para así transformarse y luego poder contribuir a la transformación de los demás, debe saber pero sobretodo, debe hacer en relación a la planeación y el direccionamiento estratégico, así como también, de manejar el tema de legislación laboral y educativa, debe ser capaz de liderar el ejercicio de autoevaluación curricular y planes de mejoramiento. 
Debe saber cómo gestionar la participación no sólo como un ejercicio de estar sino como un ejercicio de ser, debe saber cómo manejar los conflictos. Finalmente, por encima de todo lo anterior, debe ser un ejemplo en valores de sinceridad y honestidad. Aparte de ser un líder, debe promover el crecimiento, fortalecimiento y mejora de los procesos institucionales, buscar causar el logro propuesto para el año escolar, siendo desde su práctica un modelo a seguir con alto grado de responsabilidad y compromiso a través de la implementación de las virtudes de la dirección en la dimensión personal intra personal administrativa y la consolidación de las competencias como iniciativa, creatividad, autocontrol, equilibrio emocional, toma de decisiones, integridad, aprendizaje, gestión del tiempo, gestión del estrés y otros con principios éticos.

\subsection{La formación profesional y las praxis directivas en la construcción del concepto de gestión}

Para los directivos docentes participantes en esta tarea investigativa, la práctica directiva fortalecida con los diversos estudios sobre gestión han sido los aspectos que más han influido en la comprensión que tienen sobre la gestión en la escuela, elementos que se complementan con el diario vivir, el interés personal por conocer sobre la administración y la socialización de experiencias y conocimientos con otros directivos docentes.

La experiencia práctica, unida a las licenciaturas, los postgrados, los cursos, las actividades de autoaprendizaje, la participación en investigaciones y el compartir de saberes con otros directivos y profesionales pares, fortalecen las nociones directivas y contribuyen en el desarrollo de un pensamiento estratégico que les permite ejercer sus roles y cumplir sus funciones y responsabilidades.

Trabajar con y para seres humanos, les enseña a los directivos docentes a considerar la posición de cada miembro de la comunidad para proponer estrategias que incluyan las necesidades de todos los conocimientos y elementos para hacer críticas y cuestionamientos frente a las políticas que se aplican en las instituciones, la posibilidad de poner en contexto lo que se puede aportar al proyecto educativo institucional y contribuir con sus saberes para la materialización del ejercicio de la gestión. 
En su rol, los directivos han tenido la posibilidad de conocer los mecanismos de relación con otras instituciones para que la gestión sea efectiva y eficiente, es donde se retroalimenta diariamente lo que aprende a lo largo de la vida profesional, fortaleciendo su liderazgo pedagógico, los procedimientos y supervisión de las funciones, además, la evaluación de actividades que se desarrollan en el colegio, facilitando la rapidez y efectividad los procesos.

\subsection{El rol de los directivos docentes en la gestión de las instituciones educativas.}

El rol de los directivos docentes en la gestión de sus instituciones, comprende el aprendizaje constante y puesta en práctica de los conocimientos en gestionar y liderar cambios en la institución, en los currículos y participar en la toma decisiones para el mejoramiento institucional, donde se retroalimentan diariamente los conocimientos y las habilidades en todos los aspectos en la formación de un estudiante, padres de familia y la comunidad educativa, disposición, apertura, interés para el aprendizaje en este campo, actuando como facilitador de procesos en los trabajos en equipo, promoviendo la participación que le aporte a la construcción del proyecto educativo institucional.

El directivo docente tiene un papel de liderazgo y de conciliación que debe ser medido por la transparencia y la veracidad, es de liderazgo de los procesos administrativos pedagógicos académicos y propios de la comunidad, dinamizando y ajustándolos a la normatividad, canalizando las interacciones y favoreciendo el desarrollo de los procesos para el beneficio del proyecto educativo institucional.

Su tarea se centra en dirección a los objetivos de los proyectos educativos que se encuentran al alcance de cada actor educativo, debiendo enfocarse en puntos clave de los cambios y las transformaciones de las instituciones educativas, dentro de la capacidad crítica para hacer converger las políticas públicas que rigen el colegio en el contexto institucional, debe brindar un mejoramiento continuo de los procesos, para que sean permanentes, es el encargado de liberar los proyectos, planes y programas que se desarrolla en el interior de la institución, buscando el alcance de las metas y el fortalecimiento de la misma en su nivel misional. 
Es necesario rescatar el rol del directivo docente, su fortalecimiento, la ética profesional y la profundización del conocimiento de la comunidad que se encuentra trabajando en el manejo de normas, leyes y pautas para el direccionamiento estratégico.

$\mathrm{Al}$ asumir un rol directivo, se asume el reto de educar a los educadores, es decir, liderar y orientar procesos pedagógicos incentivando la creatividad para gestionar el conocimiento profesional, personal y administrativo, detectando las necesidades de formación permanente de los maestros para superar las posibilidades de conocimientos que permiten aceptar nuevos paradigmas educativos y el desarrollo de proyectos de mejoramiento para el bien común, dándole sentido a lo que se hace en la cotidianidad.

\section{Las principales conclusiones}

- El desarrollo de esta investigación, rescata la importancia de revisar la gestión en las organizaciones educativas desde la mirada de quienes hacen parte de ella y la dinamizan en la cotidianidad. En este sentido, se enfatiza en las comprensiones y los significados que los directivos docentes le imprimen a la administración y la gestión articulando las nociones humanistas que subyacen a sus tareas de dirección y que abren nuevos horizontes para concebir la gerencia, los ejercicios administrativos y las formas de organización de la escuela en el mundo actual.

- El rol y los conocimientos de los directivos docentes en sus comunidades educativas contribuyen en la ruptura de paradigmas frente a los modelos tradicionales de la gestión, respondiendo a las realidades que actualmente se viven en los planteles educativos y que exigen un pensamiento organizacional abierto a los cambios y la toma de decisiones en beneficio de la comunidad educativa.

- La gestión directiva en educación es una categoría donde confluyen diferentes conceptos que la delimitan, caracterizan y sustentan desde el quehacer de los directivos docentes, siendo ésta un campo de acción que comprende las tareas académicas, administrativas y financieras, de gestión de la comunidad y trabajo convivencial que orientan las prácticas pedagógicas y contribuyen en la dirección de todos los aspectos de la vida institucional. 
- Desde el sentir y las comprensiones de los directivos docentes, la gestión directiva hace referencia a las formas como el plantel educativo es orientado, teniendo como eje central el direccionamiento estratégico, la cultura y el clima institucionales y el liderazgo en todos y cada uno de los procesos que se llevan a cabo y que contribuyen en el éxito de la organización, su desarrollo, planeación y evaluación para el mejoramiento y el fortalecimiento.

- La gestión directiva para las organizaciones educativas escolares, vista desde la mirada de los directivos docentes, implica una definición humana del ámbito administrativo y de la gestión partiendo de los saberes, las perspectivas, las comprensiones y experiencias de los rectores y coordinadores, quiénes son los que significan este concepto en los contextos reales de sus organizaciones. De esta manera, gestionar en una institución educativa, es una tarea que implica acciones de índole administrativo, pero también de formación y sentido del humano que constituye el ser y el hacer de los planteles educativos y representan su naturaleza y su tarea de responsabilidad ante la sociedad.

\section{Bibliografía}

Álvarez, M. (2003): El equipo Directivo. Recursos Técnicos de Gestión. Editorial Laboratorio Educativo. Caracas, Venezuela. 333 p.

Ballén, A., Pulido, R. y Zúñiga, S. (2002): Abordaje hermenéutico de la investigación cualitativa. En: Ediciones Grancolombianas. Universidad La Gran Colombia. Colombia.

Decreto 1278 de 2002. Recuperado el 15 de Septiembre de 2016. Disponible En: http://www.mineducacion.gov.co/1621/articles-86102_archivo_pdf.pdf

Decreto 1850 de 2002. Recuperado el 15 de Septiembre de 2016. Disponible En: http://www.mineducacion.gov.co/1621/articles-103274_archivo_pdf.pdf 
Decreto 3020 de 2002. Recuperado el 15 de Septiembre de 2016. Disponible En: http://www.mineducacion.gov.co/1621/articles-104848_archivo_pdf.pdf

Decreto 1290 de 2009. Recuperado el 15 de Septiembre de 2009.Disponible En: http://www.mineducacion.gov.co/1621/articles187765_archivo_pdf_decreto_1290.pdf

Denzin, N. y Lincoln, Y. (2012): El campo de la investigación cualitativa. Manual de investigación cualitativa. Vol. 1. En: Gedisa Editorial. Barcelona-España.

De la Torre, G., Di Carlo, E., Florido, A., Opazo, H., Ramírez, Ch., Rodríguez, P., Sanchez, A., y Tirado, J. (2011). Teoría fundamentada o Grounded Theory. Universidad Autónoma de Madrid. España. Disponible en: http://www.academia.edu/1332754/Teor\%C3\%ADa_Fundamentada_o_Grounded_ Theory

Giroux, H. (1990): Los profesores como intelectuales. Hacia una pedagogía crítica del aprendizaje. En: Ediciones Paidós. Barcelona. España.

Giroux, H. (2001): Los profesores como intelectuales transformativos. En: Revista Docencia. Santiago. Chile. Disponible en:

http://www.revistadocencia.cl/visor.php?numeroRevista=15

Guevara, L. (2006). Gerencia social en la educación. Guía práctica para dirigir y administrar los establecimientos educativos y herramientas para implementar los sistemas de calidad y el plan de mejoramiento. Bogotá-Colombia. Ediciones Átropos.

Glaser, B. y A. Strauss (1967). The discovery of grounded theory: strategies for qualitative research. New York: Aldine Publishing Company,

Gramsci, A. (1972): Los intelectuales y la organización de la cultura. En: Ediciones Nueva Visión. Buenos Aires. Argentina.

Horkheimer, M. (2000): Crítica de la razón instrumental. En: Editorial Trotta. Madrid. España.

Lana, R (2014): La Administración Estratégica como Herramienta de Gestión. En Revista Científica Visión de Futuro. Volumen 18 Número 1. Enero a Junio.

Ley 715 de 2001. Recuperado el 12 de Septiembre de 2016. Disponible En: http://www.alcaldiabogota.gov.co/sisjur/normas/Norma1.jsp?i=4452 
Ley General de Educación (115 de 1994): Disponible en: www.alcaldiabogota.gov.co/sisjur/normas/Norma1.jsp?i=292

López, P. (2010): Variables asociadas a la gestión escolar como factores de calidad educativa. En: Estudios Pedagógicos XXXVI, No 1: 147-158. Disponible en: http://mingaonline.uach.cl/pdf/estped/v36n1/art08.pdf

Martínez, F. (2002): El cuestionario. Un instrumento para la investigación en Ciencias Sociales. En: Laertes Psicopedagogía. Barcelona. España.

Martínez, M. (2008): Epistemología y metodología cualitativa en las Ciencias Sociales. En: Trillas. México.

Martinic, S. (1997): Diseño y Evaluación de Proyectos Sociales. Herramientas para el aprendizaje. Colectivo Mexicano de Apoyo a la Niñez (COMEXANI) y Centro Juvenil Promoción Integral (CEJUV). México.

Miranda, S. y Tabares, J. (2015): Un enfoque humanista de la gestión directiva en las organizaciones educativas escolares. En: ave viajera ediciones. Bogotá (Colombia).

Noguera, Carlos: (2005). La pedagogía como "saber sometido": Un análisis del trabajo arqueológico y genealógico sobre el saber pedagógico en Colombia. En: Foucault, la pedagogía y la educación. Pensar de otro modo. Colección Pedagogía e Historia. Editorial Magisterio. Págs. 39-70.

Rincón, L. (2011): Liderazgo Orientado a la Competitividad y el Desarrollo Humano. En: UNAD, Bogotá. Escuela de Ciencias Administrativas, Contables, Económicas y de Negocios - Ecacén. 228 p.

Gorrochotegui, A. (2010): Cómo asumir el liderazgo de un centro educativo. Universidad Monteávila. Caracas. Venezuela. 150 p.

Nussbaum, M. (2005): El cultivo de la humanidad. Una defensa clásica de la reforma en la educación liberal. En: Paidós. Barcelona. España.

Pérez-Ruiz, A. (2014). Enfoques de la gestión escolar: una aproximación desde el contexto latinoamericano. Educ. Educ. 17 (2), 357-69. Doi. 10.5294/edu.2014.17.2.9. Disponible

en: http://educacionyeducadores.unisabana.edu.co/index.php/eye/article/view/3693/365 8 
Quintero, M. (2004): ¿Qué significa investigar en educación? Universidad Distrital Francisco José de Caldas. Colombia.

San Martin, D. (2013): Teoría fundamentada y Atlas.ti: recursos metodológicos para la investigación educativa. En: Revista Electrónica de Investigación Educativa Vol. 16, Núm. 1. Disponible en:

http://www.scielo.org.mx/scielo.php?pid=S1607-40412014000100008\&script=sci_arttext

Sotelo, J. (2007): Proyecto de gestión escolar e innovación Educativa. En: Libros en Red. Disponible en: https://books.google.com.co/books?id=Ui_1KErd8gYC\&printsec=frontcover\&dq= gestion+escolar\&hl=es-419\&sa=X\&ved=0ahUKEwjEl_2Qm-

HJAhUH7B4KHbmJD3UQ6AEIGjAA\#v=onepage\&q=gestion\%20escolar\&f=fals $\mathrm{e}$

Strauss, A. y Corbin, J. (2002): Bases de la investigación cualitativa. Técnicas y procedimientos para desarrollar la teoría fundamentada. En: Editorial Universidad de Antioquia. Colombia.

Tabares y Miranda (2015): Un enfoque humanista de la gestión directiva en las organizaciones educativas escolares. En: Editorial Ave Viajera. Bogotá-Colombia.

Tapiero, E. y López, L. (2006): Gestión Escolar Inteligente. Instituciones educativas Neosistémicas del Siglo XXI. Impresora Feriva S.A. Cali. Colombia. 208 p.

Taylor, S.J y Bogdan, R. (1996): Introducción A los Métodos Cualitativos de Investigación. En: Editorial Paidós, Barcelona España.

Toro, I. y Parra, R. (2010): Fundamentos epistemológicos de la investigación y la metodología de la investigación cualitativa/cuantitativa. En: Fondo Editorial Universidad EAFIT. Colombia.

Vasilachis, I. (2003): Pobres, pobreza, identidad y representaciones sociales. En: Gedisa Editorial. España.

Vasilachis, I. (2006): Estrategias de Investigación cualitativa. En: Gedisa Editorial. España.

Zapata, A. (2008): Análisis y Diseño Organizacional. De la estructura funcional a la organización vacía. Universidad del Valle. 234 p. 
Zuluaga, O. (2005): Foucault, la pedagogía y la educación. Pensar de otro modo. Colección Pedagogía e Historia. En: Editorial Magisterio. Págs. 39-70. 